\title{
IMPSO and Linear Programming-Based Energy-Efficient Cell Association Algorithm for Backhaul-Constrained Ultra-Dense Small-Cell Networks
}

\author{
Huilin Jiang $\mathbb{D}^{1,2}$ Wenxiang Zhu $\mathbb{D}^{3},{ }^{3}$ Xiang Song $\mathbb{D}^{1},{ }^{1}$ and Guilu $W u(\mathbb{D})^{4,5}$ \\ ${ }^{1}$ School of Electronic and Engineering, Nanjing Xiaozhuang University, Nanjing 211171, China \\ ${ }^{2}$ Jiangsu Key Construction Laboratory of IoT Application Technology, Wuxi, China \\ ${ }^{3}$ National Mobile Communications Research Laboratory, Southeast University, Nanjing 210096, China \\ ${ }^{4}$ Key Laboratory of Industrial Internet of Things \& Networked Control, Ministry of Education, \\ Chongqing University of Posts and Telecommunications, Chongqing 400065, China \\ ${ }^{5}$ School of Internet of Things Engineering, Jiangnan University, Wuxi 214122, China \\ Correspondence should be addressed to Wenxiang Zhu; zwx@seu.edu.cn
}

Received 7 January 2021; Revised 27 July 2021; Accepted 28 October 2021; Published 23 November 2021

Academic Editor: Venkatesan Rajinikanth

Copyright $\odot 2021$ Huilin Jiang et al. This is an open access article distributed under the Creative Commons Attribution License, which permits unrestricted use, distribution, and reproduction in any medium, provided the original work is properly cited.

This paper studies the energy efficiency optimization problem for coordinated multipoint (CoMP)-enabled and backhaulconstrained ultra-dense small-cell networks (UDNs). Energy efficiency is an eternal topic for future wireless communication networks; however, taking actual bottleneck of the backhaul link and the coordinated network architecture into consideration, it is difficult to find an effective way to improve the energy efficiency of the network. Aiming at this problem, we propose to combine cell association, subchannel allocation, backhaul resource allocation, and sleep/on of the cells together to develop an optimization algorithm for energy efficiency in UDN and then solve the formulated energy efficiency optimization problem by means of improved modified particle swarm optimization (IMPSO) and linear programming in mathematics. Simulation results indicate that nearly $13 \%$ energy cost saving and $21 \%$ energy efficiency improvement can be obtained by combining IMPSO with linear programming, and the backhaul link data rate can be improved by $30 \%$ as the number of small cells increases. From the results, it can be found that by combining IMPSO with linear programming, the proposed algorithm can improve the network energy efficiency effectively at the expense of limited complexity.

\section{Introduction}

To adapt to the ever-increasing volume of wireless traffic, industry and academic groups have paid much attention to the standardization process and theoretical research on $5 \mathrm{G}$ and beyond (B5G) and sixth generation (6G) networks [1]. To address the challenges of the future networks, a variety of advanced technologies were proposed. From the aspect of bandwidth enlargement, millimeter-wave and terahertz communication were proposed, which extended the transmission bandwidth of the wireless communication system with the help of the higher frequency band [2]. From the aspect of spectrum efficiency, state-of-the-art technologies such as massive multiple-input and multiple-output (massive MIMO) and intelligent reflective surface (IRS) were proposed to take full advantage of radio frequency spectrum $[3,4]$. Ultra-dense small-cell networks (UDNs) were proposed to further improve network throughput by deploying lower power small cells and narrowing the distance from users to their serving cells, finally enhancing the signal strength of the users $[5,6]$. In addition, to provide ever faster and higher quality of data services, fog-enabled UDNs which have communication, computing, and caching (3C) functions in small cells have been developed and have become an imperative trend for the future networks [7].

According to [8], nearly $80 \%$ of the energy consumption of information and communication technology (ICT) is caused by base station (BS) side [9]. In UDN, the energy 
consumption of a small cell is lower than that of a macrocell; however, the total energy consumption of the network would grow rapidly as the density of small cells increases, and this growth would become more rapid when fog-related functions are enabled [10]. As a result, how to improve the throughput while reducing the energy consumption of the system becomes a tough problem which challenges the deployment of UDN. This problem can be summarized as an energy efficiency optimization problem in which the energy efficiency is represented by the ratio of network throughput and the corresponding energy consumption. However, considering the fractional form of energy efficiency, how to maximize the network energy efficiency is still a challenging problem $[11,12]$.

In fog-enabled UDN, along with the increase of the density of small cells, not only the energy consumption would become higher but also the inter-cell interference (ICI) would become much more serious. Coordinated multipoint (CoMP) transmission/reception is an effective technique to mitigate ICI or transform the interfering signals to useful signals through processing the potential interference between BSs coordinately [13]. Joint transmission (JT), as one of the CoMP technologies, can unite multiple small cells and send the same data to the same user at the same time by adjusting the cell association matrix, and hence the signal-to-interference-plus-noise ratio (SINR) of the user can be improved, which finally promotes the network throughput [14]. However, in CoMP JT, the increasing number of the small cells and the simultaneous transmission of identical data to the same user will bring in additional energy consumption and backhaul burden. Therefore, how to adjust the cell association matrix in CoMP JT to balance the network throughput gain and network energy consumption to maximize the energy efficiency becomes a research hotspot. In addition, according to [15], with the increasing number of small cells, the aggregated data rate is approaching the upper limit that the backhaul link can withstand, and this aggregated data rate is proportional to the backhaul energy consumption; therefore, the corresponding backhaul energy consumption cannot be ignored either. According to the above research studies, how to allocate the data rate of backhaul link to balance the backhaul capacity gain and the corresponding energy consumption to maximize the achieved energy efficiency while the requirement of users' data rate can be met becomes an open topic. According to above reasons, how to model the energy efficiency maximization in CoMP JT while the requirement of users data rate is satisfied and considering the backhaul data rate constraint becomes an important research direction in fog-enabled UDN.

1.1. Related Works. There have been several research studies on the energy efficiency in CoMP. In [16], the authors investigated CoMP JT in the multitier UDN and analyzed the performance of network energy efficiency. In [17], the system energy efficiency maximization problem in hybridpowered heterogeneous network (HetNet) is solved by joint optimization of data and energy among coordinated BSs. In
[18], the authors focused on the CoMP JT in nonorthogonal multiple access (NOMA) and proposed a distributed power optimization approach which can obtain significant gains in energy efficiency.

Some works pay more attention to the energy-efficient cell association. In [19], the authors investigated the user-cell association problem for energy efficiency and spectral efficiency trade-off, and a Lagrange duality and game-based user-cell association algorithm was developed. The probability expressions for cell association in terms of different cell association bias values, BS density ratios, and probabilities of line of sight link are given, and the relationship between coordinated cluster and average energy efficiency is obtained in reference [20]. In [21], the authors investigated the energy consumption minimization problem by jointly optimizing the BS sleeping and cell association under the constraints of SINR and power. In [22], the authors proposed a cell association and beamforming scheme with the consideration of the local edge processing capability of the access points.

Numerical relevant studies about backhaul link constraint in UDN have been investigated. Some backhaul related research trends and challenging issues for the future networks are summarized in [23]. In [24], the authors studied the backhaul capacity and backhaul energy efficiency and pointed out the importance of cooperative and backhaul in UDN. In [25], the authors discussed the relationship between radio resource management (RRM), such as interference management, cell association, and scheduling, and backhaul link solutions and proposed a backhaul-aware RRM schemes in 5G small cells. A backhaul-aware cell selection algorithm to maximize the ergodic capacity for heterogeneous networks (HetNets) was proposed in [26].

Several studies about cell association under backhaul constraint have been investigated in recent years. In [27], the authors elaborated on cell selection policies and proposed a new cell selection algorithm considering the backhaul constraints and end-to-end performance requirements for $5 \mathrm{G}$ networks. In [28], the joint user association and content placement problem in UDN is investigated, where the delay and capacity at backhaul links are taken into consideration. In [29], the authors maximized energy efficiency in smallcell networks by jointly adjusting cell association and radio resource allocation under the constraints of quality of service and backhaul link capacity. From the aforementioned literatures, energy efficiency maximization problem for the future cooperative UDN with backhaul link constraint is rarely considered.

1.2. Motivation and Contribution. According to the discussion above, although there have been many research studies on energy efficiency in UDN, these studies have not considered the application of CoMP in future networks and the corresponding network complexity brought by it. Besides, considering the actual capacity bottleneck of the backhaul link, optimization of energy efficiency has become a major problem in UDN with CoMP. Motivated by the reasons above, in this paper, the optimization problem of energy efficiency is studied for UDN with CoMP JT, and an 
energy efficient resource allocation algorithm is proposed to solve the network complexity problem caused by CoMP under the bottleneck of the backhaul link capacity.

In detail, the energy efficiency optimization problem in the CoMP-enabled and backhaul-constrained UDN is formulated into an energy efficiency maximization problem which can be implemented by jointly adjusting the sleep/on indicator, the cell association matrix, and the backhaul link data rate, and the data rate constraints of the users and the backhaul links are taken into account. Since the sleep/on indicator, cell association, and backhaul link data rate allocation are tightly coupled with the energy consumption of both small cells and backhaul links, the closed-form solution of the problem is difficult to be obtained. Under such circumstances, we utilize the decomposition method to transform the original mixed integrate nonlinear fraction programming problem into two subproblems. The first subproblem is to minimize the energy consumption by optimizing the sleep/on indicator and cell association matrix while satisfying users' data rate requirement. This subproblem is a nonlinear zero-one programming problem which can be solved by improved modified particle swarm optimization (IMPSO). Based on the obtained optimal sleep/ on indicator and cell association matrix, the upper and lower bounds of the backhaul link data rate can be derived. Therefore, the second subproblem is to maximize the minimal achievable energy efficiency of the network by optimizing the backhaul link data rate under the obtained upper and lower bounds, which is a linear fractional programming problem. Because of the positive denominator, the second subproblem can be proved to be a quasiconvex optimization problem which can be transformed into the equivalent linear programming problem and solved by linear programming. Numerical results display that, compared with the existing algorithms, the proposed algorithm can obtain a higher network energy efficiency, higher backhaul link support, and lower energy consumption. The performance of energy efficiency shows a decreasing trend with the increasing number of small cells due to the multiplied energy consumption of the small cells and the increasing interference among cells. In addition, although the backhaul link energy consumption of the proposed algorithm is higher than the existing algorithms, total energy consumption of the network is much lower.

The main contributions are as follows:

(i) Different from the existing works, energy efficiency optimization problem in UDN under the scenario of CoMP JT and limited backhaul link capacity is considered, where more than one cell can be selected as the cooperative serving cells for a user while the data rate requirement of users should be guaranteed under the backhaul link constraint.

(ii) A mixed-integer nonlinear programming problem for joint optimization of the sleep/on indicator, the cell association matrix, and the backhaul link data rate to maximize the energy efficiency of network under the constraints of backhaul links and users' data rate requirement is formulated. Because of the coupling relationship among the variables, the focused problem is challenging to be solved.

(iii) To solve the formulated complex problem, decomposition method is adopted to transform the original problem into two subproblems. The first is to minimize the energy consumption of the network by controlling the sleep/on status of the cells and the serving cells of the users. The second is to maximize the minimum energy efficiency by allocating backhaul link data rate.

(iv) A joint optimization algorithm based on the combination of IMPSO and linear programming is proposed to solve the focused energy efficiency optimization problem. Besides, to enable the proposed algorithm, the upper and lower bounds of the backhaul link constraint are derived.

(v) Simulations are implemented to validate the effectiveness of the proposed algorithm in terms of the network energy efficiency. The impact of the number of users and small cells on the energy efficiency, energy consumption, and backhaul link data rate is evaluated and discussed.

The organization of the rest of this paper is as follows. A brief introduction on the system model is given in Section 2 . In Section 3, a network energy efficiency maximization problem with data rate constraints of users and backhaul link in UDN with CoMP JT is formulated. An energy-efficient cell association and backhaul link optimization algorithm is proposed in Section 4. The transformed energy consumption minimization subproblem with users' data rate requirement is formulated as a nonlinear zero-one programming problem, and an IMPSO-based cell association algorithm is proposed in Section 4.1. In Section 4.2, we propose a linear programming-based backhaul link data rate allocation algorithm to solve the second transformed subproblem. Results of the numerical simulation are presented and analyzed in Section 5, and the conclusion is presented in Section 6.

\section{System Model}

Consider the downlink transmission of an orthogonal frequency division multiplexing (OFDM) technology-assisted and fog-enabled UDN. As shown in Figure 1, the entire network consists of $N_{C}$ small cells, $N_{S}$ switches, several users, forward/backhaul links, and a center unit. Small cells are equipped with communication and caching functions. The forward links are wireless links between small cells and users, and the backhaul links are the wired links between switches and small cells. Each user $u$ has its own data rate requirement $\widetilde{R}_{u}$. Based on the location of the requested contents of the users, users in the network are divided into two sets. The first set contains the users whose required contents have been cached in the small cells. The second set contains the users whose requested contents have not been cached in the small cells, denoted as $\mathscr{U}=\left\{u_{1}, u_{2}, \ldots, u_{N_{U}}\right\}$, where $N_{U}$ is the number of users in $\mathscr{U}$. Since the required 


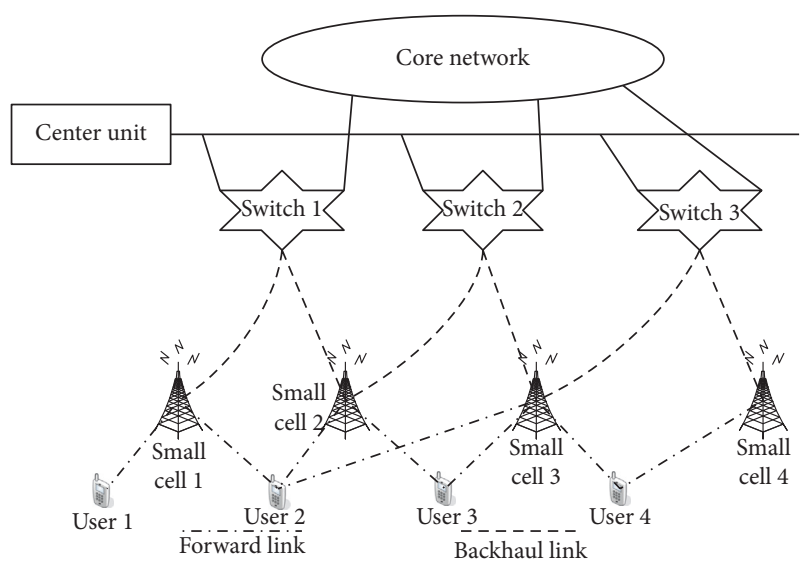

Figure 1: System model.

contents have not been cached in the small cells, users in $\mathcal{U}$ have to fetch contents from the center unit through switches and backhaul links. The switches have a common quota data rate. The resource allocation on switches and small cells is conducted by the core network. Suppose the network uses a dedicated portion of resources to serve the users whose required contents have been cached in the small cells; in this paper, resource allocation to users in $\mathcal{U}$ is focused. The set of small cells and switches is $\mathscr{C}=\left\{c_{1}, c_{2}, \ldots, c_{N_{C}}\right\}$ and $\mathcal{S}=\left\{s_{1}, s_{2}, \ldots, s_{N_{S}}\right\}$, respectively. The content data requested by the users in $\mathcal{U}$ are firstly delivered from the center unit to the switches connected to the core network and then sent to the small cells through backhaul links. Finally, the required contents are delivered to users.

In this paper, we denote the open access mode as the access criterion and the users can select one or multiple small cells as their serving cells by CoMP JT technology. The total frequency bandwidth shared among all small cells to serve the users in set $\mathcal{U}$ is $W$, which is equally allocated to $L$ subchannel whose set is $\mathscr{L}=\{1,2, \ldots, L\}$. The subchannels of a small cell are allocated the same transmission power [30]. The cell association matrix between small cell and users can be expressed as $\mathscr{A}=\left\{a_{1,1}, a_{1,2}, \ldots, a_{N_{C} * N_{U}}\right\}$, in which $a_{c, u} \in \mathscr{A}$ is the cell association index. If user $u$ is associated with small cell $c, a_{c, u}$ is equal to 1 ; otherwise, $a_{c, u}$ is equal to 0 .

Denote the transmission power of each subchannel in small cell $c$ as $p_{c}^{t}, c \in \mathscr{C}$, the reference signal received power (RSRP) $p_{c, u}$ of user $u$ from small cell $c$ is

$$
p_{c, u}=p_{c}^{t} G_{c, u}, \quad c \in \mathscr{C}, u \in \mathcal{U},
$$

where $G_{c, u}$ represents the average channel gain which consists of antenna gain, shadow fading, and path loss between small cell $c$ and user $u$. In fact, a real system should avoid frequent handover when the users' serving cell is selected [27]. Compared with the time scale of small-scale fading, the cell association adjustment in a large time scale is much slower. This is because the cell association adjustment has a coupled relationship with the serving cell decision of the users. Therefore, like references [27, 28], in this paper, we do not consider the small-scale fading case when we calculate the average channel gain. The SINR of user $u$ is calculated by

$$
\gamma_{c, u}=\frac{\sum_{c \in \mathscr{C}} a_{c, u} p_{c}^{t} G_{c, u}}{\sum_{i \in \mathscr{C}} p_{i}^{t} G_{i, u}-\sum_{c \in \mathscr{C}} a_{c, u} p_{c}^{t} G_{c, u}+\sigma^{2}}
$$

where $\sigma^{2}$ is the noise power of each subchannel. The average data rate of each subchannel of user $u$ is

$$
R_{c, u}=\frac{W}{L} * \log _{2}\left(1+\frac{\sum_{c \in \mathscr{C}} a_{c, u} p_{c}^{t} G_{c, u}}{\sum_{i \in \mathscr{C}} p_{i}^{t} G_{i, u}-\sum_{c \in \mathscr{C}} a_{c, u} p_{c}^{t} G_{c, u}+\sigma^{2}},\right) .
$$

Considering that each user has its own data rate requirement $\widetilde{R}_{\mathcal{u}}$, the required number of subchannels for user $u$ is $n_{c, u}=\left\lceil\widetilde{R}_{u} / R_{c, u}\right\rceil$, where $\lceil x\rceil$ is the minimum integer which is not less than $x$. According to the linear approximation energy consumption model given by Earth Project, the energy consumption of small cell $c$ is

$$
p_{c}= \begin{cases}p_{c}^{h}+\Delta_{c} p_{c}^{t} \sum_{u \in \mathscr{U}} \sum_{l \in \mathscr{L}} \tilde{a}_{c, u}^{l}, & N_{c}>0, \\ p_{c}^{s}, & N_{c}=0,\end{cases}
$$

where $p_{c}, p_{c}^{h}$, and $p_{c}^{s}$ denote the energy consumption of small cell $c$, the hardware energy consumption of small cell $c$ in ideal state, and the hardware energy consumption of small cell $c$ in sleep state, respectively. $\Delta_{c}$ is the reciprocal of power amplifier efficiency of small cell $c$ and $\tilde{a}_{c, u}^{l} \in \tilde{\mathscr{A}}=\left\{\tilde{a}_{1,1}^{1}, \widetilde{a}_{1,1}^{2}, \ldots, \widetilde{a}_{N_{C}, N_{U}}^{N_{L}}\right\}$ is an association index indicating whether the subchannel $l$ of small cell $c$ is allocated to user $u$. The backhaul energy consumption is

$$
\tilde{p}_{s}=\sum_{c \in \mathscr{C}} \beta_{c, s} x_{c, s}, \quad \forall s \in \mathcal{S},
$$

where $\beta_{c, s}$ is the energy consumption slope and $x_{c, s} \in \mathscr{X}=$ $\left\{x_{1,1}, x_{1,2}, \ldots, x_{N_{C}, N_{S}}\right\}$ is the data rate of backhaul link between small cell $c$ and switch $s$.

Considering the data rate on the forward/backhaul links and the corresponding energy consumption of the backhaul links together, energy efficiency of the network can be calculated by

$$
\eta_{E E}=\frac{\sum_{c \in \mathscr{C}} \min \left\{\sum_{u \in \mathcal{U}} \sum_{l \in \mathscr{L}} \widetilde{a}_{c, u}^{l} R_{c, u}, \sum_{s \in \mathcal{S}} \beta_{c, s} x_{c, s}\right\}}{\sum_{c \in \mathscr{C}}\left[\left(1-\delta_{c}\right) p_{c}^{s}+\delta_{c}\left(p_{c}^{h}+\Delta_{c} p_{c}^{t} \sum_{u \in \mathcal{U}} \sum_{l \in \mathscr{L}} \widetilde{a}_{c, u}^{l}\right)\right]+\sum_{s \in \mathcal{S}} \sum_{s \in \mathcal{S}} \beta_{c, s} x_{c, s}},
$$


where $\delta_{c} \in \mathscr{B}=\left\{\delta_{1}, \delta_{2}, \ldots, \delta_{N_{C}}\right\}$ is the cell sleep/on indicator. When the number of users is zero in the small cell $c$, small cell $c$ can be turned into sleep state, i.e., $\delta_{c}=0$; otherwise, $\delta_{c}=1$.

\section{Problem Formulation}

Aiming at finding optimal $\mathscr{A}, \tilde{A}, \mathscr{B}$, and $\mathscr{X}$ to maximize the achieved energy efficiency of the network with CoMP JT while satisfying the constraints of cell association relationship, users' data rate requirements, and backhaul link data rate, we formulate the optimization problem as $P 1$ :

$$
\begin{aligned}
& P 1: \max _{\mathscr{A}, \widetilde{A}, \mathscr{B}, \mathscr{X}} \eta_{E E}=\frac{\sum_{c \in \mathscr{C}} \min \left\{\sum_{u \in \mathscr{U}} \sum_{l \in \mathscr{L}} \tilde{a}_{c, u}^{l} R_{c, u}, \sum_{s \in \mathcal{S}} \beta_{c, s} x_{c, s}\right\}}{\sum_{c \in \mathscr{C}}\left[\left(1-\delta_{c}\right) p_{c}^{s}+\delta_{c}\left(p_{c}^{h}+\Delta_{c} p_{c}^{t} \sum_{u \in \mathscr{U}} \sum_{l \in \mathscr{L}} \tilde{a}_{c, u}^{l}\right)\right]+\sum_{s \in \mathcal{S}} \sum_{s \in \mathcal{S}} \beta_{c, s} x_{c, s}}, \\
& \text { s.t. } \widetilde{a}_{c, u}^{l} \in\{0,1\}, \delta_{c} \in\{0,1\}, a_{c, u} \in\{0,1\}, \quad \forall c \in \mathscr{C}, \forall u \in \mathscr{U}, \forall l \in \mathscr{L} \text {, } \\
& x_{c, s} \geq 0, \quad \forall c \in \mathscr{C}, \forall s \in \mathcal{S}, \\
& a_{c, u} \geq \tilde{a}_{c, u}^{l}, \quad \forall c \in \mathscr{C}, \forall u \in \mathscr{U}, \forall l \in \mathscr{L}, \\
& \sum_{c \in \mathscr{C}} a_{c, u} \geq 1, \quad \forall u \in \mathscr{U} \\
& \sum_{u \in \mathscr{U}} \sum_{l \in \mathscr{L}} \tilde{a}_{c, u}^{l} \leq \delta_{c} L, \sum_{c \in \mathscr{C}} \sum_{l \in \mathscr{L}} \tilde{a}_{c, u}^{l} R_{c, u} \geq \widetilde{R}_{u}, \quad \forall u \in \mathscr{U}, \forall c \in \mathscr{C}, \\
& \sum_{s \in \mathcal{S}} x_{c, s} \geq \sum_{u \in \mathscr{U}} a_{c, u} \widetilde{R}_{u}, \quad \forall c \in \mathscr{C} \\
& \sum_{c \in \mathscr{C}} x_{c, s} \leq \tilde{q}, \quad \forall s \in \mathcal{S}
\end{aligned}
$$

where $\widetilde{q}$ is the quota data rate of each switch. The energy efficiency of network can be taken as the objective function (7a) and the optimization variables are the cell association matrix, the subchannel occupied cell association matrix, cell sleep/on indicator, and backhaul link data rate. (7b) and (7c) denote the range of the cell association index, the subchannel occupied cell association index, cell sleep/on indicator, and backhaul link data rate, respectively. (7d) indicates the relationship between the cell association index and the subchannel occupied cell association index. (7e) indicates that users can be associated with not less than one small cell due to CoMP JT technology. (7f) indicates that each small cell should meet the sum of subchannel constraint and each user should meet the users' data requirement. $(7 \mathrm{~g})$ indicates that the allocated backhaul link data rate of each small cell should not be less than the users' data rate requirement of each small cell. (7h) is quota data rate constraint of each switch.

\section{Energy-Efficient Cooperative Cell Association and Backhaul Link Optimization Algorithm}

Similar to [24], in this paper, we consider the case that the backhaul link data rate cannot meet the forward link data rate of small cell $c$. That is to say, $\sum_{u \in \mathscr{U}} \sum_{l \in \mathscr{L}} \widetilde{a}_{c, u}^{l} R_{c, u}>\sum x_{c, s}$. So, the optimal solution of optimization problem $P 1^{\text {s }}$ \&an be obtained when the forward link data rate of small cells is not less than the corresponding backhaul link data rate. Therefore, the original problem $P 1$ can be transformed into P2:

$$
P 2 \max \widetilde{\eta}_{E E}=\frac{\sum_{c \in \mathscr{C}} \sum_{s \in \mathcal{S}} x_{c, s}}{\sum_{c \in \mathscr{C}}\left[\left(1-\delta_{c}\right) p_{c}^{s}+\delta_{c}\left(p_{c}^{h}+\Delta_{c} p_{c}^{t} \sum_{u \in \mathscr{U}} \sum_{l \in \mathscr{L}} \tilde{a}_{c, u}^{l}\right)\right]+\sum_{s \in \mathcal{S}} \sum_{c \in \mathscr{C}} \beta_{c, s} x_{c, s}},
$$

s.t. $(7 b)-(7 h)$, 


$$
\sum_{u \in \mathscr{U}} \sum_{l \in \mathscr{L}} \tilde{a}_{c, u}^{l} R_{c, u} \geq \sum_{s \in \mathcal{S}} x_{c, s}, \quad \forall c \in \mathscr{C}
$$

$$
\sum_{u \in \mathscr{U}} n_{c, u} a_{c, u} \leq \delta_{c} L, \quad \forall c \in \mathscr{C}
$$

where $(8 \mathrm{c})$ is the added extra constraint of $P 1$ representing rate. From $P 2$, we found out that the formulated optimization problem is very difficult which cannot be solved by the conventional algorithm. Therefore, we propose an energy-efficient cooperative cell association and backhaul link optimization (EECCABLO) algorithm to solve it.

Considering the difficulty of $P 2$, we transform it into two subproblems. The first subproblem is a cell association problem to minimize energy consumption with users' data rate constraints, which can be represented by

$$
P 3 \min \psi_{E C}=\sum_{c \in \mathscr{C}}\left[\left(1-\delta_{c}\right) p_{c}^{s}+\delta_{c}\left(p_{c}^{h}+\Delta_{c} p_{c}^{t} \sum_{u \in \mathcal{U}} n_{c, u} a_{c, u}\right)\right],
$$

$$
\text { s.t. }(7 b),(7 d) \text {, }
$$

where (9c) is the subchannel constraint of small cell. $P 3$ is a zero-one nonlinear programming problem and the optimization variables are cell association matrix and sleep/on indicator.

Considering the calculation complexity and the optimization variable scale of problem (9a 9b 9c), the optimal solution is difficult to be found by employing traditional methods. Under such circumstances, using penalty function, we further transform (9a) into the following nonconstrained optimization problem:

$$
\begin{aligned}
P 4 \min \psi_{E C}=\sum_{c \in \mathscr{C}} & {\left[\left(1-\delta_{c}\right) p_{c}^{s}+\delta_{c}\left(p_{c}^{h}+\Delta_{c} p_{c}^{t} \sum_{u \in \mathscr{U}} n_{c, u} a_{c, u}\right)\right]+\sigma\left(\sum_{u \in \mathscr{U}}\left[\max \left\{0, \sum_{c \in \mathscr{C}} a_{c, u}-1\right\}\right]^{\alpha}\right) } \\
& +\sigma\left(\sum_{c \in \mathscr{C}}\left[\max \left\{0, \sum_{u \in \mathscr{U}} n_{c, u} a_{c, u}-\delta_{c} L\right\}\right]^{\alpha}+\sum_{c \in \mathscr{C}} \sum_{u \in \mathscr{U}}\left|a_{c, u}^{2}-a_{c, u}\right|^{\mu}+\sum_{c \in \mathscr{C}}\left|\delta_{c}^{2}-\delta_{c}\right|^{\mu}\right),
\end{aligned}
$$

where $\sigma$ is the penalty factor and $\alpha, \mu \in N^{+}$denote the coefficients of constraint violations. Then, we propose an IMPSO-based cell selection algorithm to solve this problem.

\subsection{Improved Modified Particle Swarm Optimization-Based} Cell Association Algorithm. As a kind of metaheuristic algorithm [31], particle swarm optimization (PSO) is a swarm intelligence optimization algorithm where there are many particles and each particle has its own behavioral characteristics, such as position, velocity, and so on. To obtain the objective function value, the fitness position of a particle needs to be recognized and it is also a potential solution of the problem. The adjustment scale of the potential solution can be expressed by the velocity of a particle. By the velocities of all particles, each particle can update its position information.

Considering the discrete optimization variables, to avoid dropping into fake optimal solution, having a fast convergence speed and guaranteeing the optimality, rounded integer, the local search mechanism, tent-based chaotic mapping, and multiple restart are introduced to the MPSO, where the framework is just like PSO. The solution set of optimization problem (9a) consists cell association matrix and sleep/on indicator, and the corresponding fitness value is the energy consumption.
Taking particle $i$ as an example, the solution set is denoted as $\mathcal{S}^{i}=\left[\mathscr{A}^{i}, \mathscr{B}^{i}\right], i=1,2, \ldots, \zeta$, where $\zeta$ is the total number of particles. $\mathscr{A}^{i}=\left\{a_{1,1}^{i}, a_{1,2}^{i}, \ldots, a_{N_{C}, N_{U}}^{i}\right\}$ is the cell association matrix set, and $\delta_{c} \in \mathscr{B}=\left\{\delta_{1}, \delta_{2}, \ldots, \delta_{N_{C}}\right\}$ is the sleep/on indicator set. Mark the whole adjustment scale of cell association matrix and sleep/on indicator of particle $i$ as a $\quad N_{U} \times\left(N_{C}+1\right) \quad$ dimensional dataset $\mathscr{V}^{i}=\left[V_{1,1}^{i}, V_{1,2}^{i}, \ldots, V_{1, N_{U}}^{i}, \quad V_{2,1}^{i}, \ldots, V_{N_{C}, N_{U}}^{i}\right.$, $\left.V_{N_{C}+1,1}^{i}, \ldots, V_{N_{C}+1, N_{U}}^{i}\right]$, where the adjustment scale range is $[-1,1]$. The MPSO-based energy efficiency resource allocation and cell association algorithm in detail is summarized as follows.

In the initialization process, the system parameters, including restart time $\left(t_{s}=0\right)$, iteration time $(t=0)$, maximum iteration time $\left(t_{-} \max \right)$, and maximum restart time $\left(t_{s^{-}} \max \right)$, are all initialized. The initial solution set and the adjustment scale set with all particles are selected randomly. The fitness value of particle $i$ at iteration $t$ is given by

$$
\psi_{E C}^{i}(t)=\psi_{E C}\left(\mathcal{S}^{i}(t)\right)
$$

The personal best solution and the global best solution are denoted as the important characters when each particle updates its velocity. The best fitness value ever found by the particle is defined as the personal best solution. Similarly, the best solution found among all the particle is defined as the 
global best solution. The personal best solution of particle $i$ at iteration $t$ is given by

$$
\delta_{S}^{i}(t)=\underset{\delta^{i}(\tau)}{\arg \max } \psi_{E C}\left(\mathcal{S}^{i}(\tau)\right), \quad \tau=0,1, \ldots, t,
$$

where $\mathcal{S}_{s}$ is the personal best solution and $\tau=0$ is the initialization phase. The global best solution at iteration $t$ can be computed as

$$
\mathcal{S}_{g}(t)=\underset{\mathcal{S}_{s}^{i}(t)}{\arg \max } \psi_{E C}\left(\mathcal{S}_{s}^{i}(t)\right), \quad i=1,2, \ldots \zeta
$$

where $\mathcal{S}_{g}$ is the global best solution.

Then, at iteration $t+1$, the $j$ th variable of the solution set of particle $i$ updates its velocity $V_{j}^{i}(t+1)$ according to the personal best solution and the global best solution as follows:

$$
\begin{aligned}
V_{j}^{i}(t+1)= & \omega(t) V_{j}^{i}(t)+c_{1} r_{1, j}(t)\left[S_{j}^{i}(t)-S_{s, j}^{i}(t)\right] \\
& +c_{2} r_{2, j}(t)\left[S_{j}^{i}(t)-S_{g, j}(t)\right], \quad i=1,2, \ldots, \zeta ; j=1,2, \ldots, N_{C} \times\left(N_{U}+1\right),
\end{aligned}
$$

where $\omega$ is the inertia weight factor, which is a key parameter that can impact the convergence performance of PSO [32].

Considering that the tent-based chaotic mapping has an advantage in chaotic ergodicity and stochastic property, we take it into the updated of inertia weight factor to speed up the convergence speed, and the updated formulation can be expressed as

$$
\omega(t+1)= \begin{cases}2 \omega(t), & 0 \leq \omega(t) \leq 1 / 2, \\ 2(1-\omega(t)), & 1 / 2 \leq \omega(t) \leq 1,\end{cases}
$$

where $S_{s, j}^{i}(t)$ and $S_{g, j}(t)$ represent the $j$ th value of the personal best solution and the global best solution at iteration $t$, respectively. $c_{1}$ and $c_{2}$ are the learning factors which correspond to the personal best solution and the global best solution, respectively. In addition, the variables of $r_{1, j}$ and $r_{2, j}$ have the uniform distribution interval $[0,1]$.

From formula (15), we find out that the state of inertia weight factor, the personal best solution, the global best solution, and the random variables of the last iteration have influence on the updated adjustment scale. Thereafter, the particle $i$ updates its solution at iteration $t+1$ according to

$$
\mathcal{S}^{i}(t+1)=\operatorname{INT}\left(\mathcal{S}^{i}(t)+\mathscr{V}^{i}(t+1)\right),
$$

where INT $(x)$ expresses rounded integer for $x$. To avoid falling into fake optimal solution, the MPSO adopts the local search mechanism in the case where $\omega(t)$ approaches to zero. The specific updating method on the global best solution and the adjustment scale is given by

$$
\begin{aligned}
& \mathcal{S}_{g}(t+1)=\operatorname{INT}\left(\mathcal{S}_{g}(t)+\omega(t) \mathscr{V}_{g}(t)+\mathscr{Z}(t)\right), \\
& \mathscr{V}_{g}(t+1)=\omega(t) \mathscr{V}_{g}(t)+\mathscr{Z}(t),
\end{aligned}
$$

where $\mathscr{Z}(t)=\left[Z_{1}(t), Z_{2}(t), \ldots, Z_{N_{C}}(t), \ldots, Z_{N_{C} \times\left(N_{U}+1\right)}(t)\right]$ is the newly added random variable set. Range of values of each component of $\mathscr{Z}(t)$ is $\left[-r_{\theta}(t), r_{\theta}(t)\right]$. Here, $r_{\theta}(t)$ is the local search radius, and it is determined by the ratio of the number of consecutive successful iterations $n_{s}$ and consecutive failure iterations $n_{f}$.

In order to avoid the current optimal solution falling into the fake local or global optimal solution, $r_{\theta}(t)$ should be adjusted timely to find a better solution when the ratio exceeds the predefined threshold $n_{t h}$; otherwise, $r_{\theta}(t)$ should be reduced to shrunk the selected range. Based on the aforementioned analysis, the local search radius is defined as

$$
r_{\theta}(t+1)=\left\{\begin{array}{cl}
\gamma r_{\theta}(t), & n_{s} / n_{f}>n_{t h} \\
\frac{r_{\theta}(t)}{\gamma}, & n_{s} / n_{f}<n_{t h} \\
r_{\theta}(t), & \text { otherwise }
\end{array}\right.
$$

where $\gamma$ is the radius regulation factor, $r_{\theta}(0)=1, n_{t h}=1.2$, and $\gamma=2 . n_{s}$ and $n_{f}$ are initialized as 1 , and the value will be reinitialized when the global best solution is updated. Additionally, the multirestart process is introduced in the proposed algorithm to guarantee to find the best solution as the global best solution among all the multirestart solutions. When the iteration time $t>t_{-}$max or the restart time $t=t_{s^{-}}$max, the algorithm will stop running.

4.2. Linear Programming-Based Backhaul Link Data Rate Optimization Algorithm. Based on the optimal cell association matrix and sleep/on indicator obtained by solving $P 3$, we can divide the small cell set $\mathscr{C}$ into two subsets $\{\overline{\mathscr{C}}, \underline{\mathscr{C}}\}$ which are the turned on small cell set $\overline{\mathscr{C}}=\left\{c \in \mathscr{C} \mid \delta_{c}=1\right\}$ and the turned off small cell set $\mathscr{C}=\left\{c \in \mathscr{C} \mid \delta_{c}=0\right\}$, respectively. The set of user associated with small cell $c$ can be represented as $\xi_{c}=\left\{u \in \mathcal{U} \mid a_{c, u}=1\right\}$. For the nonempty set $\xi_{c} \neq \varnothing$, considering the data rate requirement of users in $\xi_{c} \neq \varnothing$ and the constraint of (8c), the lower bound $\underline{R}$ and upper bound $\bar{R}$ of data rate of small cell $c$ can be calculated by $\underline{R}_{c}=\sum_{u \in \xi_{c}} \widetilde{R}_{u}$ and $\bar{R}_{c}=\underline{R}_{c}+\left(L-\sum_{u \in \xi_{c}} n_{c, u}\right) \max _{u \in \epsilon} R_{c, u}$, respectively. For the empty set $\xi_{c}=\varnothing, \underline{R}_{c}=\bar{R}_{c} \stackrel{u}{=} \epsilon_{\hat{\theta}}$. Based on the above calculation, we can further transform the minimum achievable maximum energy efficiency as

$$
P 4 \bar{\eta}_{E E}=\max \frac{\sum_{c \in \overline{\mathscr{C}}} \sum_{s \in \mathcal{S}} x_{c, s}}{\sum_{c \in \overline{\mathscr{C}}}\left[p_{c}^{h}+\Delta_{c} p_{c}^{t} L\right]+\sum_{s \in \mathcal{S}} \sum_{c \in \overline{\mathscr{C}}} \beta_{c, s} x_{c, s}},
$$

$$
\text { s.t. }(7 b),(7 d) \text {, }
$$




$$
\underline{R}_{c} \leq \sum_{s \in \mathcal{S}} x_{c, s} \leq \bar{R}_{c}, \quad \forall c \in \mathscr{C},
$$

where (19a) is the objective function of maximum achievable energy efficiency while the optimization variables refer to backhaul links' data rate and (19c) indicates that the backhaul link data rate of each small cell should meet the upper and lower bound constraints.

From the formulated optimization problem $P 4$, we can find that it is a linear fraction programming problem about $x_{c, s}$. Considering that the denominator of $\bar{\eta}_{E E}$ is the network energy consumption, the domain of $\bar{\eta}_{E E}$ about $x_{c, s}$ is positive. The objective function is quasiconvex (in fact, quasilinear), so linear fractional program is a quasiconvex optimization problem. Assuming that there exists a solution set that can meet the constraints, we can transform $P 4$ into the equivalent linear programming $P 5$ :

$$
\begin{gathered}
P 5 \min -\bar{\eta}_{E E}=-c^{T} y-\mathrm{d} z, \\
\text { s.t. } K y-h z \leq 0, \\
H y-\bar{R} z \leq 0, \\
\underline{R} z-H y \leq 0, \\
e^{T} y+f z=1, \\
z \geq 0,
\end{gathered}
$$

where $x=\sum_{c \in \overline{\mathscr{C}}} \sum_{s \in \mathcal{S}} x_{c, s}, e^{T}=\sum_{s \in \mathcal{S}} \sum_{c \in \overline{\mathscr{C}}} \beta_{c, s}, f=\sum_{c \in \overline{\mathscr{C}}}\left[p_{c}^{h}+\right.$ $\left.\Delta_{c} p_{c}^{t} L\right], y=x /\left(e^{T} x+f\right), z=1 /\left(e^{T} x+f\right), c^{T}=I_{1, N_{C} * N_{S}}$, and $h=\widetilde{q} I_{N_{S}, 1}$. In addition, $K_{N_{C}, N_{C} \times N_{S}}$ and $H_{N_{C}, N_{C}} * N_{S}$ are coefficient matrices. If $(y, z)$ is feasible in $P 5$, with $z \neq 0$, then $x=y / z$ is the feasible backhaul link data rate solution which can be solved by linear programming [33] and the corresponding energy efficiency can be obtained.

\section{Simulation Results and Performance Analysis}

In this section, numerical results are conducted to validate the proposed EECCABLO algorithm by comparing it with the existing resource allocation algorithms. The first one is the conventional cell association and backhaul link resource allocation scheme where each user can only be associated with one cell named EECS. The remaining algorithms are EEUB and EELB which are obtained by equally allocating the upper bound and lower bound backhaul link data rate under the EECCABLO cell association scheme, respectively. The simulation parameters are shown in Table 1 according to the Earth Project and 3rd Generation Partnership Project (3GPP). The simulation scenario will be given out subsequently: several small cells are clustered deployed in the network, $2 / 3$ of the users are cluster distributed in the coverage of small cells, and the remaining $1 / 3$ of the users are randomly distributed in the network. To obtain the required results, we will operate multiple independent experiments in the simulation progress.
5.1. The Energy Efficiency Performance with the Increasing Number of Small Cells under Different Algorithms. In this section, the performance of energy efficiency for the case of 60 users and $2 \mathrm{Mbps}$ of the users' data rate requirement is investigated. The simulation results with the increasing number of small cells under different algorithms are shown in Figure 2. It can be seen from the figure that with the increasing number of the small cells, the maximized minimum achievable energy efficiency performance is in a decreasing trend. That is because as the number of small cells increased, the network energy consumption is also in an increasing trend and the network inter-cell interference becomes much complex. In addition, considering that EECCABLO adopts CoMP JT, it needs less subchannels to meet users' data rate requirement than EECS, so as to reduce energy consumption and enhance the maximized minimum achievable energy efficiency. Since there is no optimization in the backhaul link data rate allocation in EEUB and EELB, their energy efficiency performance is lower than that of EECCABLO and EECS. Besides, considering the randomness of backhaul link energy consumption, there exists fluctuation in EEUB.

5.2. The Energy Efficiency Performance with the Increasing Number of Users under Different Algorithms. In this section, we investigate the energy efficiency with the increasing number of users under different algorithms. The number of small cells and switches is 15 and 6, respectively. The users' data rate requirement is $1 \mathrm{Mbps}$. In Figure 3, we can see that the energy efficiency is in an increasing trend with the increasing number of users when the number of users is less. As the number of users further increases, the energy efficiency becomes flat. This is because there are enough resources to serve users when the number of users is small. As the number of users further increases, the total amount of users' data rate requirement is also increased and the number of occupied subchannels is increased. Since the total backhaul link resources are constant, the network energy consumption and backhaul link data rate of EECCABLO become flat by optimizing backhaul link data rate. Hence, the maximized minimal achieved energy efficiency becomes flat. The energy efficiency of EEUB increases at first and then it decreases. This is because, at beginning, there are enough resources to serve users and the backhaul link data rate gain is higher than network energy consumption. Then, as the number of users further increases, the required subchannels are increased and the corresponding energy consumption is also increased. However, in EEUB, the backhaul link data rate is not optimized, so the maximized minimal energy efficiency is in a decreasing trend. Since the backhaul link data rate of EELB is the sum of users' data rate requirement, the backhaul link data rate and network energy consumption become flat. Therefore, the simulation result of EELB becomes flat.

5.3. The Backhaul Link Data Rate Performance with the Increasing Number of Small Cells under Different Algorithms. The backhaul link data rate performance with the increasing number of small cells under different algorithms is 
TABLE 1: Simulation parameters.

\begin{tabular}{lc}
\hline Parameter & Value \\
\hline The transmission power of small cell $p_{c}^{t}(\mathrm{~mW})$ & 130 \\
Bandwidth $W(\mathrm{MHz})$ & 10 \\
Carrier frequency $(\mathrm{GHz})$ & 2 \\
The power consumption of small cell in ideal and sleep mode $(\mathrm{mW})$ & 6800 and 4300 \\
The reciprocal of power amplifier efficiency of small cell & 4 \\
Antenna gain of small cell $(\mathrm{dBi})$ & 5 \\
Shadow standard deviation of small cell $(\mathrm{dB})$ & 10 \\
Path loss of small cell $R(\mathrm{~km})$ & $140.7+36.7 \log 10(\mathrm{R}) \mathrm{dB}$ \\
The number of subchannels & 50 \\
The number of switches & 4 \\
Quota of switch $\widetilde{q}$ (Mbps) & 50 \\
Noise power density per subchannel $\sigma^{2}(\mathrm{dBm} / \mathrm{Hz})$ & -174 \\
Backhaul link power consumption slope $\beta(\mathrm{W} / \mathrm{Mbps})$ & Random $[0.1,0.2]$ \\
Coefficients of constraint violations $\alpha, \mu$ & 2 \\
\hline
\end{tabular}

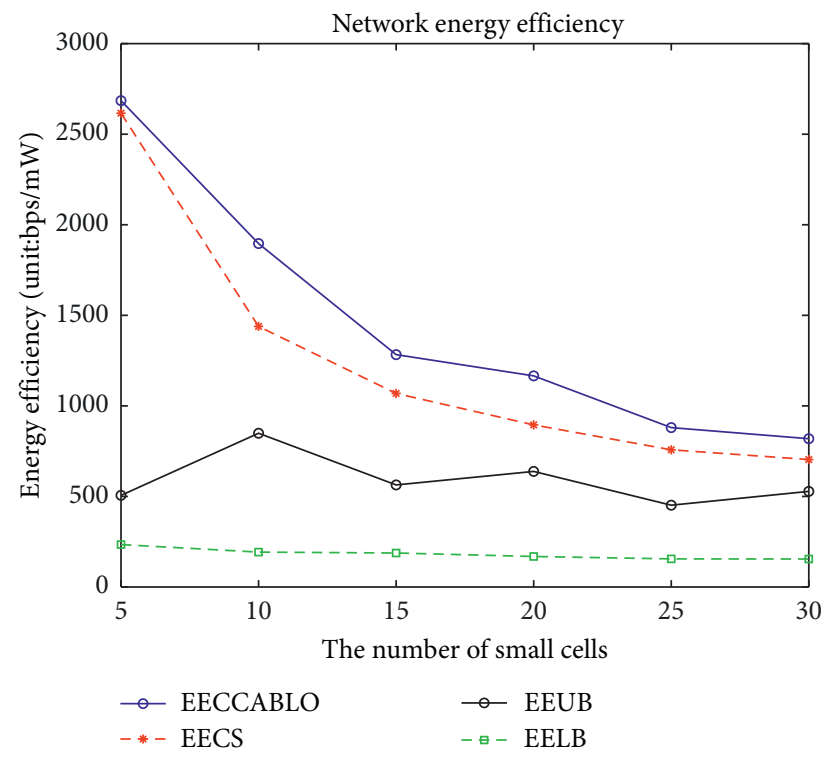

FIGURE 2: The performance of energy efficiency with the increasing number of small cells.

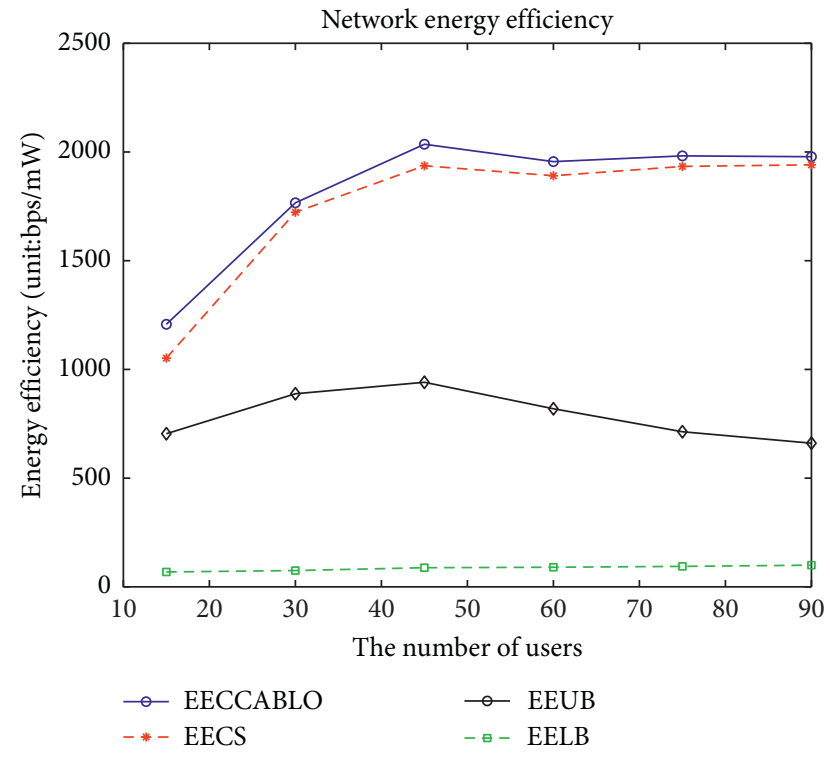

FIGURE 3: The performance of energy efficiency with the increasing number of users. 
investigated, and the simulation results are shown in Figure 4 . The simulation results are calculated by the sum of the backhaul link data rate allocated to the small cells. $\mathrm{RD}$ is short of all the users' required data rate. In the simulation result, the proposed EECCABLO algorithm and EECS algorithm both can meet the users' data rate requirement. Since CoMP JT is adopted by EECCABLO, the backhaul link data rate of EECCABLO is higher than that of EECS. In addition, considering that the quota data rate of each switch is constant, the sum data rate of all the switches is fixed. Since the optimization object function is maximized minimal achieved energy efficiency and the backhaul link energy consumption is proportional to backhaul link data rate, it is not best to allocate the total amount of backhaul link data rate to all small cells. For this reason, the sum of the backhaul link data rate is not equal to the sum of the quota data rate of all switches. Since RD is only relative to the users' data rate requirement and user's data rate is a random value between 0.1 and $R$, the RD is a random value.

\subsection{The Backhaul Link Data Rate Performance with the In-} creasing Number of Users under Different Algorithms. From the simulation results of backhaul link data rate with the increasing number of users in Figure 5, we can see that the backhaul link data rate of EECCABLO and EECS increases firstly and then becomes flat. This is because, at the beginning, the number of users is small, and it does not take too much wireless resources to meet users' data rate requirement. With the increasing number of users, more wireless resources are required and corresponding backhaul link data rate also increases. Because the total quota data rate of all switches is fixed, the resource allocated is finite. In addition, considering that the optimization object function is maximized minimal energy efficiency, the algorithm should jointly consider the backhaul link data rate and energy consumption instead of allocating all quota data rate to small cells. RD increases with the number of users, and the backhaul link data rate of EECCABLO and EECS is increased.

\subsection{The Backhaul Link Energy Consumption Performance with} the Increasing Number of Small Cells and Users under Different Algorithms. The energy consumption of backhaul link with the increasing number of small cells and users is investigated in this section. From Figures 6 and 7, we can see that EECCABLO algorithm has a higher energy consumption than EECS. That is because the energy consumption of backhaul link is proportional to the backhaul link data rate, which means that higher backhaul link data rate leads to higher backhaul link energy consumption. In addition, by jointly considering Figure 4 and the random energy consumption slope of the backhaul link, the backhaul link energy consumption is different even though it has the same backhaul link data rate.

5.6. The Network Energy Consumption Performance with the Increasing Number of Small Cells and Users under Different Algorithms. In this section, we investigate the network

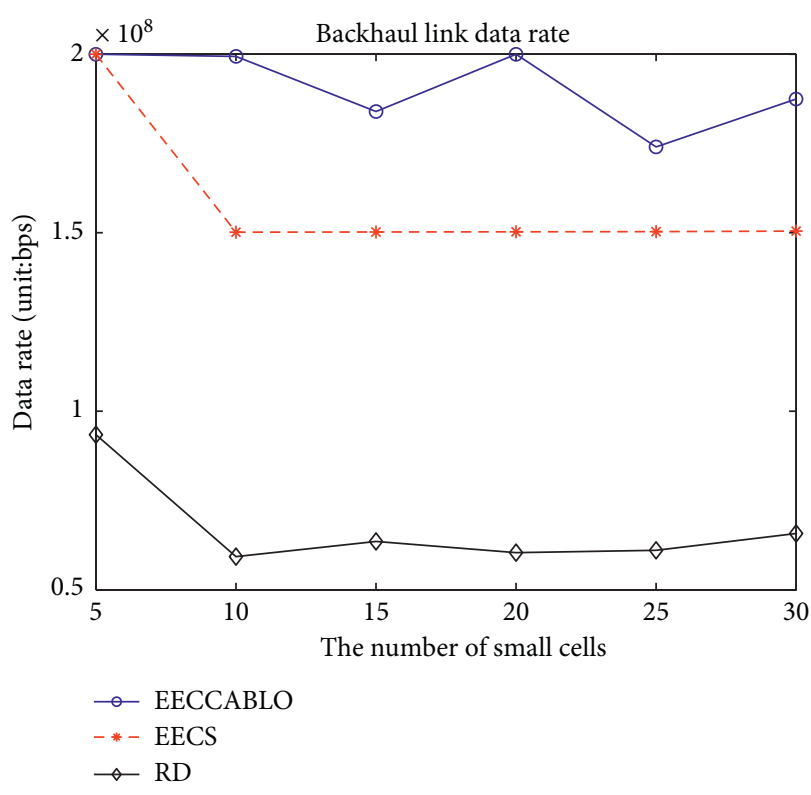

FIgURE 4: The performance of backhaul link data rate with the increasing number of small cells.

energy consumption performance with the increasing number of small cells and users under different algorithms. The network energy consumption includes two parts which are the energy consumption of small cells and backhaul link, respectively. From the simulation results in Figure 8, we find out that the energy consumption is in an increasing trend with the number of small cells increased. Although the energy consumption of backhaul link of EECCABLO algorithm is higher than that of EECS algorithm, the energy consumption of small cells side is much lower. That is because, although CoMP JT requires more small cells to transmit data to the user simultaneously, it occupies fewer subchannels by optimizing the cell association, sleep/on indicator, and backhaul link data rate allocation. Therefore, in a summary, the total network energy consumption of EECCABLO algorithm is less than that of EECS algorithm. As the number of users increases, the performance gap between the two algorithms is relatively small; therefore, the simulation results are summarized in Table 2 instead of simulation curve. The first row is the number of users, the first column denotes the algorithms, and the rest is the corresponding energy consumption. From the results, we can see that the energy consumption of EECCABLO is lower than that of EECS. This is because, as the number of users increases, more resources are required to serve users. Considering EECCABLO adopts CoMP JT, although much energy consumption is consumed in backhaul link, few subchannels are required to serve users and total energy consumption decreases. In addition, the total energy consumption is little when the number of users is little. As the number of users increases, the network energy consumption increases firstly and then becomes flat. This is because, when the number of users is little, there are enough resources to meet users' data rate requirement and improve the minimal achievable energy efficiency. So, the 


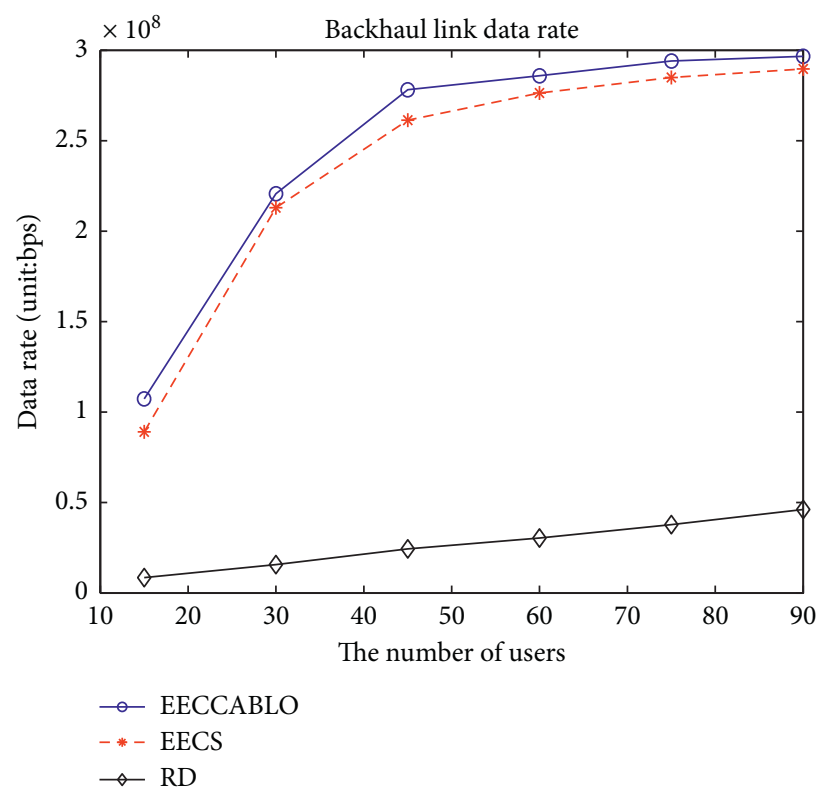

FIGURE 5: The performance of backhaul link data rate with the increasing number of users.

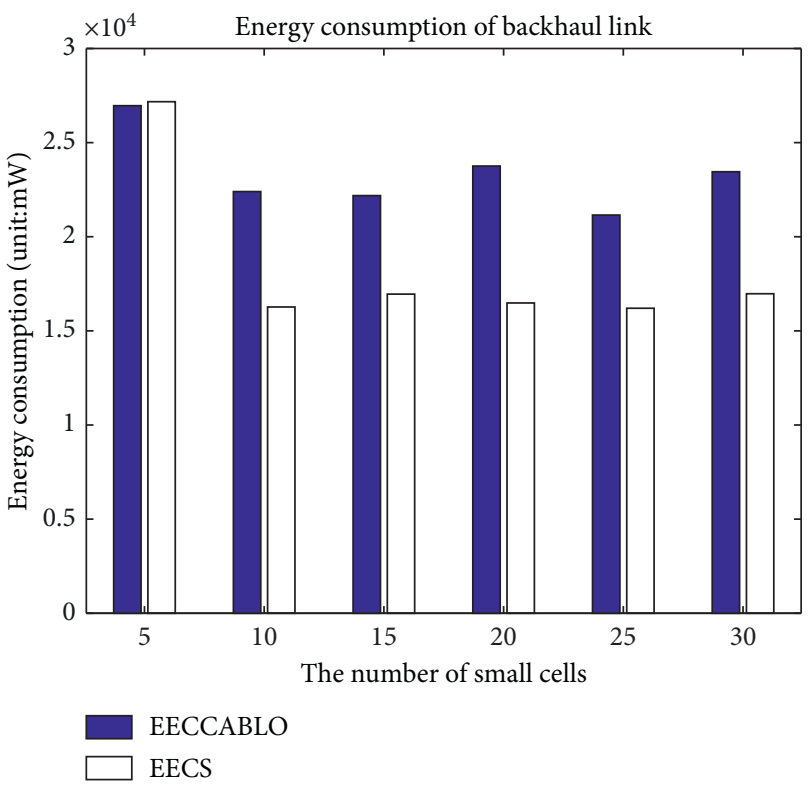

FIGURE 6: The performance of backhaul link energy consumption with the increasing number of small cells.

energy consumption increases fast. However, since the quota data rate of switches is finite, as the number of users further increases, the finite resource should firstly satisfy

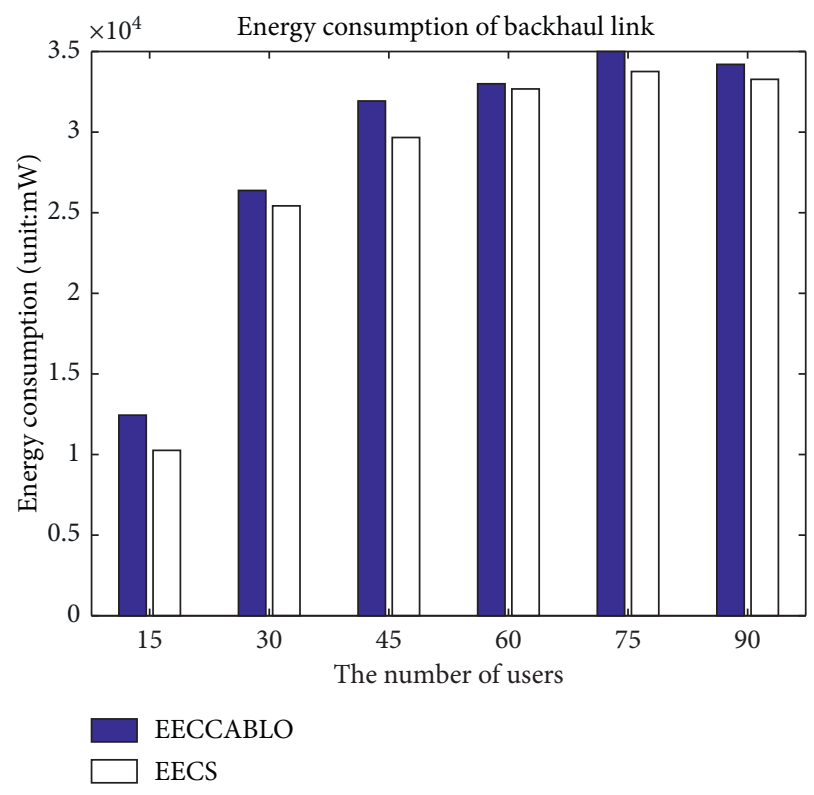

FIGURE 7: The performance of backhaul link energy consumption with the increasing number of users.

the date rate of all users and improve the minimal achievable energy efficiency secondly. Therefore, the network energy consumption becomes flat. 


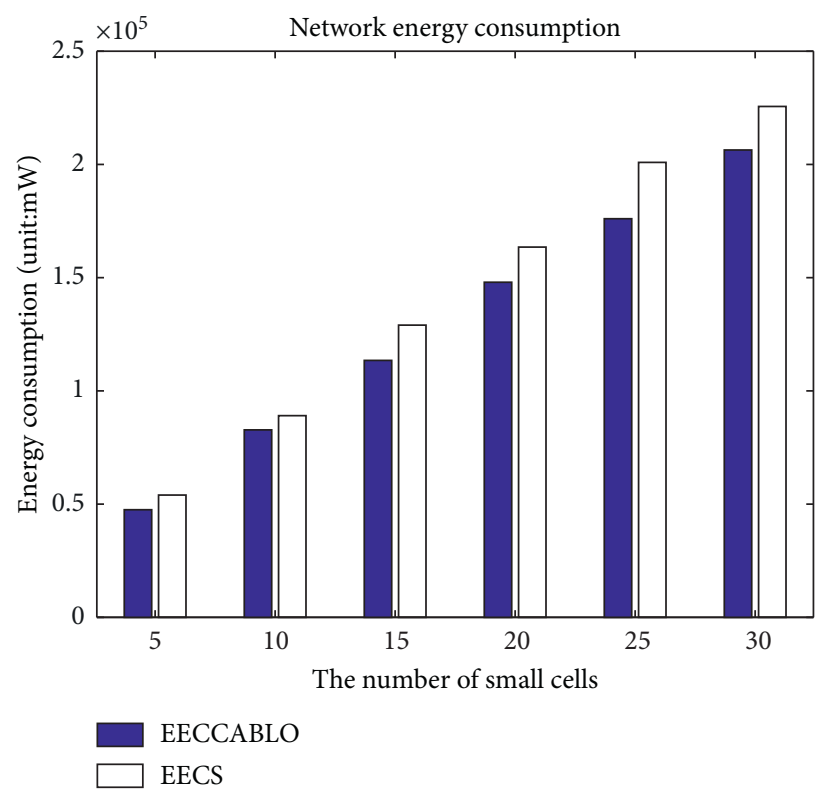

FIGURE 8: The performance of network energy consumption with the increasing number of small cells under different algorithms.

TABLE 2: The performance of network energy consumption with the increasing number of users.

\begin{tabular}{lcccccc}
\hline & 15 & 30 & 45 & 60 & 75 & 90 \\
\hline $\begin{array}{l}\text { EECCABLO } \\
(\mathrm{mW})\end{array}$ & 72780 & 96960 & 104580 & 113000 & 113460 & 115840 \\
EECS (mW) & 72900 & 97020 & 104660 & 113260 & 113600 & 116040 \\
\hline
\end{tabular}

\section{Conclusions}

In this paper, use case of the backhaul-constrained UDN where the backhaul link data rate cannot meet the requirement of the forward link was considered, and the network energy efficiency optimization problem under CoMP JT was handled by jointly optimizing the cell association, cell sleep/on, and backhaul link resource allocation of the network. Considering the tightly coupled relationship of the optimization variables and the nonconvexity of the formulated optimization problem, we transformed the original problem into two subproblems and proposed an IMPSO-based cell association algorithm and an LP-based backhaul link data rate allocation algorithm to solve them, respectively. Numerical simulations were conducted, and the results showed that, compared with existing algorithms, the proposed algorithm had a higher energy efficiency, higher network throughput, and lower energy consumption. In addition, the proposed algorithm obtained a higher backhaul link data rate than that of the existing algorithms while guaranteeing the users' data rate requirement.

The main contribution of this paper is in providing a novel resource allocation algorithm for improving the energy efficiency of UDN under complex CoMP scenarios and backhaul link constraint. Different from the existing studies, mixed-integer nonlinear programming problem for joint optimization of sleep/on indicator, cell association, and backhaul data rate was formulated. After that, the decomposition method was employed to split the problem into two. Finally, IMPSO and linear programming are adopted to solve the two subproblems. Benefitting from the proposed algorithm, the energy consumption of UDN can be saved by $13 \%$ and the energy efficiency of UDN can be improved by $21 \%$. At the same time, more than $30 \%$ improvement in backhaul link data rate can be obtained.

In the future, the proposed algorithm can also be extended to the scenario of partial cache and slicing networks. Furthermore, different caching capabilities and service types can be taken into consideration.

\section{Data Availability}

The data used to support the findings of this study are available from the corresponding author upon request.

\section{Conflicts of Interest}

The authors declare that they have no conflicts of interest.

\section{Acknowledgments}

This research was supported in part by the National Natural Science Foundation of China (no. 61801227), Qinglan Project of Jiangsu Province (nos. QLGC2021 and QLGC2020), Future Network Scientific Research Fund Project (nos. FNSRFP-2021-YB-28 and FNSRFP-2021-YB29), Open Research Fund of Jiangsu Key Construction Laboratory of IoT Application Technology (no. 19WXWL04), University-Industry Collaborative Education Program (no. 202101220012), and Key Laboratory of Industrial Internet of Things and Networked Control, Ministry of Education (no. 2019FF09). 


\section{References}

[1] J. Zhu, M. Zhao, S. Zhang, and W. Zhou, "Exploring the road to 6G: ABC-foundation for intelligent mobile networks," China Communications, vol. 17, no. 6, pp. 51-67, 2020.

[2] C.-X. Wang, J. Huang, H. Wang, X. Gao, X. You, and Y. Hao, "6G wireless channel measurements and models: trends and challenges," IEEE Vehicular Technology Magazine, vol. 15, no. 4, pp. 22-32, 2020.

[3] S. Verma, S. Kaur, M. A. Khan, and P. Sehdev, "Toward green communication in 6G-enabled massive Internet of Things," IEEE Internet of Things Journal, vol. 8, no. 7, pp. 5408-5415, 2021.

[4] Y. Pan, K. Wang, C. Pan, H. Zhu, and J. Wang, "UAV-assisted and intelligent reflecting surfaces-supported terahertz communications," IEEE Wireless Communications Letters, vol. 10, no. 6, pp. 1256-1260, 2021.

[5] L. Yu, Z. Liu, M. Wen et al., "Sparse code multiple access for 6G wireless communication networks: recent advances and future directions," IEEE Communications Standards Magazine, pp. 1-13, 2021, early access.

[6] B. Hu, F. Zuo, C. a. Wang, and S. Chen, "A proactive selection method for dynamic access points grouping in user-centric UDN," China Communications, vol. 18, no. 4, pp. 153-165, 2021.

[7] M. Peng, T. Q. S. Quek, G. Mao, Z. Ding, and C. Wang, "Artificial-intelligence-driven fog radio access networks: recent advances and future trends," IEEE Wireless Communications, vol. 27, no. 2, pp. 12-13, 2020.

[8] P. Li, Y. Shen, F. Sahito, Z. Pan, and X. You, "BS sleeping strategy for energy-delay tradeoff in wireless-backhauling UDN," Science China Information Sciences, vol. 62, no. 4, pp. 42303:1-42303:17, 2019.

[9] R. K. A. Saha, "Theoretical framework toward realizing spectral and energy efficiencies of $6 \mathrm{G}$ mobile networks," in Proceedings of the IEEE 92nd Vehicular Technology Conference (VTC2020-Fall), pp. 1-7, Victoria, BC, Canada, December 2020.

[10] K. Guo, M. Sheng, J. Tang, T. Q. S. Quek, and Z. Qiu, "Hierarchical offloading for delay-constrained applications in fog RAN," IEEE Transactions on Vehicular Technology, vol. 69, no. 4, pp. 4257-4270, 2020.

[11] Z. Dong, J. Wei, X. Chen, and P. Zheng, "Energy efficiency optimization and resource allocation of cross-layer broadband wireless communication system," IEEE Access, vol. 8, pp. 50740-50754, 2020.

[12] G. Yenihayat and E. Karaşan, "Downlink data rate, energy and spectral efficiency distribution in heterogeneous networks with cell-edge located small cells," Wireless Networks, vol. 1, pp. 1-9, 2019.

[13] M. S. J. Solaija, H. Salman, A. B. Kihero, M. I. Saglam, and H. Arslan, "Generalized coordinated multipoint framework for 5G and beyond," IEEE Access, vol. 9, pp. 72499-72515, 2021.

[14] L. Liu, Y. Q. Zhou, and A. V. Vasilakos, "Time-domain ICIC and optimized designs for $5 \mathrm{G}$ and beyond: a survey," Science China Information Sciences, vol. 62, pp. 14-41, 2019.

[15] B. Li, Y. Dai, Z. Dong, E. Panayirci, H. Jiang, and H. Jiang, "Energy-efficient resources allocation with millimeter-wave massive MIMO in ultra dense HetNets by SWIPT and CoMP,"
IEEE Transactions on Wireless Communications, 2021, Early Access.

[16] S. Mukherjee, D. Kim, and J. Lee, "Base station coordination scheme for multi-tier ultra-dense networks," IEEE Transactions on Wireless Communications, 2021, early access.

[17] S. Euttamarajah, Y. H. Ng, and C. K. Tan, "Energy-efficient joint power allocation and energy cooperation for hybridpowered comp-enabled HetNet," IEEE Access, vol. 8, pp. 29169-29175, 2020.

[18] M. S. Ali, E. Hossain, A. Al-Dweik, and D. I. Kim, "Downlink power allocation for CoMP-NOMA in multi-cell networks," IEEE Transactions on Communications, vol. 66, no. 9, pp. 3982-3998, 2018.

[19] S. Cetinkaya and H. Arslan, "A distributed user-cell association for spectral and energy efficiency tradeoff in massive MIMO UDHNs," in Proceedings of the IEEE 30th Annual International Symposium on Personal, Indoor and Mobile Radio Communications (PIMRC), pp. 1-6, Istanbul, Turkey, September 2019.

[20] S. Fang, G. Chen, X. Xu, S. Han, and J. Tang, "Millimeter-wave coordinated beamforming enabled cooperative network: a stochastic geometry approach," IEEE Transactions on Communications, vol. 69, pp. 1068-1079, 2021.

[21] X. Xu, C. Yuan, W. Chen, X. Tao, and Y. Sun, “Adaptive cell zooming and sleeping for green heterogeneous ultradense networks," IEEE Transactions on Vehicular Technology, vol. 67, no. 2, pp. 1612-1621, 2018.

[22] T. H. L. Dinh, M. Kaneko, E. H. Fukuda, and L. Boukhatem, "Energy efficient resource allocation optimization in fog radio access networks with outdated channel knowledge," IEEE Transactions on Green Communications and Networking, vol. 5, no. 1, pp. 146-159, 2021.

[23] E. Yaacoub and M.-S. Alouini, "A key 6G challenge and opportunity-connecting the base of the pyramid: a survey on rural connectivity," Proceedings of the IEEE, vol. 108, no. 4, pp. 533-582, 2020.

[24] R. Thakur, S. N. Swain, and C. S. R. Murthy, "An energy efficient cell selection framework for femtocell networks with limited backhaul link capacity," IEEE Systems Journal, vol. 12, no. 2, pp. 1969-1980, 2018.

[25] Q. Zhang, W. Ma, Z. Feng, and Z. Han, "Backhaul-capacityaware interference mitigation framework in $6 \mathrm{G}$ cellular Internet of Things," IEEE Internet of Things Journal, vol. 8, no. 12, pp. 10071-10084, 2021.

[26] H. Zhang, Y. Chen, and Z. Han, "Explicit modelling and performance analysis of cell group selection with backhaulaware biasing," IEEE Wireless Communications Letters, vol. 8, no. 1, pp. 273-276, 2019.

[27] S. Ranjan, P. Chaporkar, P. Jha, and A. Karandikar, "Backhaul-aware cell selection policies in 5G IAB networks," in Proceedings of the IEEE Wireless Communications and Networking Conference (WCNC), pp. 1-6, Nanjing, China, January 2021.

[28] W. Teng, M. Sheng, K. Guo, and Z. Qiu, "Content placement and user association for delay minimization in small cell networks," IEEE Transactions on Vehicular Technology, vol. 68, no. 10, pp. 10201-10215, 2019.

[29] S. Tong, Y. Liu, M. Cheriet, M. Kadoch, and B. Shen, "UCAA: user-centric user association and resource allocation in fog computing networks," IEEE Access, vol. 8, pp. 10671-10685, 2020 . 
[30] P. Li, H. L. Jiang, Z. W. Pan, and X. H. You, "Energy-delay tradeoff in ultra-dense networks considering BS sleeping and cell association," IEEE Transactions on Vehicular Technology, vol. 67, pp. 734-751, 2018.

[31] N. Razmjooy, V. Estrela, H. Loschi, and W. Farfan, "A comprehensive survey of new meta-heuristic algorithms," Recent Advances in Hybrid Metaheuristics for Data Clustering, Wiley, Hoboken, NJ, USA, 2019.

[32] H. Jiang, Z. Pan, N. Liu, and X. You, "LD-IMPSO based power adjustment algorithm for eICIC in QoS constrained hyper dense HetNets," Wireless Personal Communications, vol. 88, no. 2, pp. 111-131, 2016.

[33] G. B. Dantzig, Linear Programming and Extensions, Princeton University Press, Princeton, NJ, USA, 1963. 